Article

\title{
Performance Analysis of Hybrid Protocol Based AF EH Relaying over Asymmetric Fading Channels
}

\author{
Xutao Sheng ${ }^{1}$, Guangyue Lu ${ }^{1, *}$, Liqin Shi ${ }^{2}$ and Yinghui Ye ${ }^{2}(\mathbb{D}$ \\ 1 Shaanxi Key Laboratory of Information Communication Network and Security, \\ Xi'an University of Posts and Telecommunications, Xi'an 710121, China; sheng_xutao@163.com \\ 2 School of Telecommunications Engineering, Xidian University, Xi'an 710071, China; \\ liqinshi27@gmail.com (L.S.); yeyinghui1991@stu.xidian.edu.cn (Y.Y.) \\ * Correspondence: gylu@xupt.edu.cn
}

Received: 22 January 2019; Accepted: 1 February 2019; Published: 4 February 2019

\begin{abstract}
Simultaneous wireless information and power transfer is a practicable solution to encourage energy-constrained relay nodes to cooperate with the source to transmit information to the destination. In this paper, we study the outage performance of hybrid protocol based amplify-and-forward (AF) relay networks over asymmetric fading channels, where the source-relay link and the relay-destination link are subjected to Rician fading and Rayleigh fading, respectively. In particular, we derive the lower bound of outage probability and the upper bound of outage capacity based on a high signal-to-noise ratio approximation, respectively. We further investigate the effects of various system parameters, such as the parameters of hybrid protocol, the target rate, and the Rician $K$-factor, on the investigated network. It is shown that a good selection of parameters of hybrid protocol is of significance to improve system capacity, and that a larger Rician factor is desirable in the investigated network.
\end{abstract}

Keywords: simultaneous wireless information and power transfer; relay network; outage performance; hybrid protocol; asymmetric fading

\section{Introduction}

Internet of things (IoT) allows massive wireless devices, e.g., low power wireless sensor nodes, to access wireless networks and communicate with each other via a parallel access channel or multiple-access channel [1-4]. Typically for a wireless sensors network, wireless sensor nodes usually send their data to the cluster head $(\mathrm{CH})$ and then the $\mathrm{CH}$ uploads the collected data to the sink node $[5,6]$. To this end, there are many issues that need to be well addressed such as the decision fusion design fusion center (e.g., $\mathrm{CH}$ ), resource management, the reliability-guaranteed power supply for wireless IoT devices, and so on [1-7]. Among them, the reliability-guaranteed power supply for wireless IoT devices attracted much attention recently, and simultaneous wireless information and power transfer (SWIPT) [8] has been proposed and devoted to the solution of this issue. On the other hand, wireless relaying technology has been recognized as an important technology in IoT networks [1] since it is highly beneficial in the communication range, the energy efficiency and the system capacity. For example, in a wireless sensor network, the $\mathrm{CH}$ node may send data to the sink node and the link between the cluster and the node may suffer severe fading. In this case, a relay is needed to assist their transmission and enhance its performance. However, the wireless IoT devices are usually energy-constrained and unwilling to serve as a relay in practice, due to the extra energy consumption for relaying operations. Therefore, SWIPT can be integrated to the relay network in order to achieve a tradeoff between power supply and information processing from different perspectives, e.g., energy efficiency [9] and outage performance $[8,10]$. 
Until now, there have many reports [10-20] regarding amplify-and-forward (AF)/decode-and-forward (DF) relay networks with SWIPT. The authors of [10] proposed two energy harvesting (EH) relaying protocols, i.e., time switching (TS) and power splitting (PS), and investigated the corresponding outage performance of an AF relay network. The outage performance behavior of an AF relay network in the presence of a direct link between the source and the destination was also analyzed in [12]. Exploiting a heterogeneous time allocation protocol, the authors in [13] studied the outage performance and ergodic performance of PS based DF relay networks. Different from the prior works, the authors in [14] derived the closed-form expression of outage capacity for TS based multi-relay AF networks, where the source-relay and relay-destination links are modelled as Rayleigh fading and Gaussian fading, respectively. Later, the outage performance analyses of PS/TS based AF/DF relay networks over different fading channels, e.g., log-normal and Nakagami- $m$, were also presented [15-18]. Other works on the integration of the SWIPT based relay networks and other advanced technologies, such as the non-orthogonal multiple access and the cognitive radio, can also be found in $[19,20]$. These works mentioned above have laid a solid foundation for the understanding of PS/TS protocol in relay networks, and have also revealed their advantages and disadvantages. For example, the PS protocol achieves a higher capacity than the TS protocol at high input signal-to-noise ratio (SNR), while TS is more desirable in terms of both achievable capacity and hardware complexity at relative low SNR or high target rate $[8,10,13]$.

To explore the advantages of both PS and TS protocols, the authors of [21] introduced a novel hybrid protocol, which takes both PS and TS into considerations. Compared with PS and TS, the hybrid protocol is more general and achieves a higher capacity. Therefore, it is meaningful to study the hybrid protocol carefully and some contributions [21-23] have been reported regarding the application of the hybrid protocol in the AF relay network. In [21], the optimal hybrid protocol was developed to maximize the system capacity by optimizing the parameters of hybrid protocol, i.e., TS ratio and PS ratio, based on the instantaneous channel state information, subject to the 'harvest-use' scheme. A similar work [22] was further discussed based on the 'harvest-store-use'scheme instead of 'harvest-use' scheme. By means of stochastic theory, the authors of [23] derived the expression of outage capacity for hybrid protocol based AF relay networks. The derived results provide valuable insights into the impact of important system parameters especially for the PS ratio and TS ratio. These works [21-23] were based on the Rayleigh fading channel, which may be an inappropriate model for the source-relay link. The reasons are as follows. In the IoT networks, the IoT device (referring to the source) requires an immediate EH relay to assist its transmission if the direct link between the source and the destination is blocked due to the shielding effect caused by obstacles. Further, owing to the relatively short wireless power transfer distance $[8,24,25]$, it is reasonable to assume that the EH relay is close to the source and far from the destination. In this case, the source has a good channel to the relay, inferring that there possibly exist both the line-of-sight (LOS) path and the scattered multipath for the source-relay link, while the channel of relay to destination may be poor. As pointed out in [26], the Rician fading is one of optimal candidates to model the wireless channel above since the Rician fading includes line-of-sight (LOS) path and the scattered multipath. Moreover, the Rayleigh fading is a special case of Rician fading. This is to say, the Rician fading can be reduced to the Rayleigh fading by adjusting its parameters. Accordingly, it is reasonable to consider the asymmetric fading channels, where the source-relay and relay-destination links experience Rician fading and Rayleigh fading, respectively.

To the best of our knowledge, the outage performance (note that the outage probability and outage capacity are critical performance metrics in SWIPT based relay networks, and hence there exist many works $[10-15,23]$ on the outage performance anlaysis) of hybrid protocol over asymmetric fading channels has not been studied and this motivates this work. Note that the performance analysis over asymmetric fading channels is much challenging than the symmetric fading channels since the Rician fading is more complexity than the Rayleigh fading and the Rayleigh fading is a special case of Rician fading. 
The main contributions are as follows.

- Different from the existing works [21-23] with a focus on the outage performance of a hybrid protocol over symmetric fading channels, we consider a more practical asymmetric fading channels, where the source-relay and relay-destination links experience Rician fading and Rayleigh fading, respectively. In particular, we derive the expressions for the bound of outage probability and outage capacity based on the high SNR approximation and the Gaussian-Chebyshev quadrature.

- Numerical results are presented to verify the derived expression, and study the impacts of various network parameters on the outage capacity of hybrid protocol based relay networks. This provides some insights in selecting the parameters of hybrid protocol, i.e., TS and PS ratios, under different relay network settings. It can also be revealed that the parameters of hybrid protocol have a significant impact on the outage capacity, and that a larger Rician factor achieves a higher outage capacity. Besides, we compare the outage capacity achieved by the hybrid protocol, PS protocol and TS protocol. It is shown that the hybrid protocol can be reduced to the PS or TS mode at some system settings so as to reduce the cost of complexity while maintaining the required outage capacity.

The rest of this paper organizes as follows. In Section 2, we introduce the system model and working flow. The outage probability and outage capacity are derived in Section 3 , and numerical simulations are presented to verify the derived results and find the insights on the choosing parameters of hybrid protocol in Section 4. Finally, the conclusion of this paper is given in Section 5. Besides, all the used notations in this paper have been summarized in Table 1.

Table 1. Summary of Notations.

\begin{tabular}{cc}
\hline Notations & Meaning \\
\hline$I_{0}(x)$ & The zeroth order modified Bessel function of the first kind \\
$\operatorname{Prob}(\cdot)$ & Probability of a random event \\
$\min (\cdot, \cdot)$ & The minimal function \\
$Q(\cdot, \cdot)$ & The first-order Marcum $Q$ function \\
$C_{\mathrm{Euler}}$ & Euler's constant and its value is 0.5772156649 \\
$\operatorname{Ei}(\cdot)$ & The exponential integral function \\
$\Gamma(\cdot, \cdot)$ & Incomplete gamma function \\
\hline
\end{tabular}

\section{System Model and Working Flow}

\subsection{System Model}

This paper investigates an AF relay network [10], in which an energy-constrained EH relay (R) is employed to assist the communications between the source node (S) and the destination node (D) under the guidance of a hybrid protocol [21,23], as shown in Figure 1 (note that this relay model has been widely used in many state-of-art works [21,23], and that our work can be extended to more complex scenario with multiple sources or multiple relays. For the case with multiple relays, we can select a best relay to maximize the end-to-end SNR via relay selection schemes. If a single relay is selected, the scenario with multiple relays is reduced to our considered scenario. For the case with multiple sources, according to [7], all the sources can operate under orthogonal frequency multiple access scheme so that transmission interference is avoided. However, the performance analysis of this scenario will bring new challenge, which is beyond of the scope of this paper).The parameters $\alpha$ and $\beta$ are the PS and TS ratios, and the detailed information on the hybrid protocol can be found in Section II of [21]. We assume that only both the source and the destination have enough power supply, and that the 'harvest-use' scheme is employed to encourage the relay to complete the cooperate function. Also, all nodes operate in a half-duplex mode. Note that the application of full-duplex mode [9] in 
the relay node can be used to improve the performance, which is an interesting problem and can be studied in our future work. All channels are assumed to be independent and modeled as quasi-static block-fading. Recognizing the existence of a strong LOS path of source-relay link, we assume that the source-relay link and the relay-destination link follow Rician fading and Rayleigh fading, respectively.
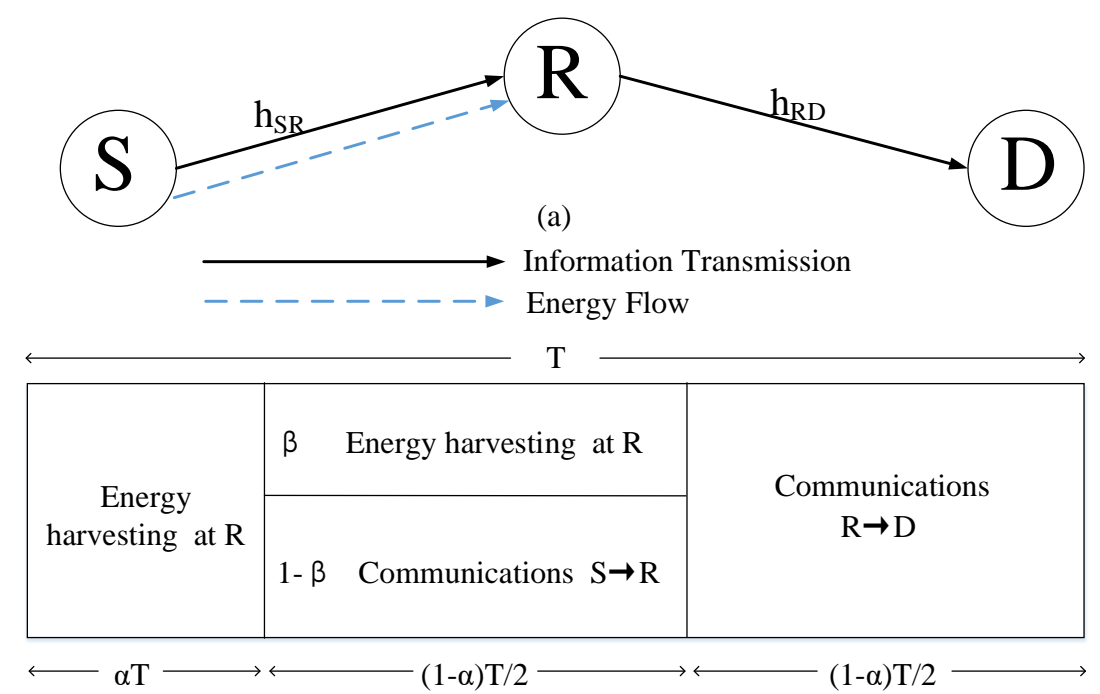

(b)

Figure 1. (a) System network; (b) an illustration of hybrid protocol.

\subsection{Analytical Model}

\subsubsection{Source-Relay Transmission}

The received signal at the relay node can be given as

$$
y_{\mathrm{R}}(t)=\sqrt{P_{\mathrm{S}} d_{\mathrm{SR}}^{-m}} h_{\mathrm{SR}} s(t)+n_{a}^{[\mathrm{R}]}(t)
$$

where $P_{S}$ is the transmitted power from the source; $s(t)$ is the normalized information signal from the source; $d_{\mathrm{SR}}^{-m}\left|h_{\mathrm{SR}}\right|^{2}$ is the adopted path-loss model including the large-scale path loss and small-scale fading; $m$ and $d_{\mathrm{SR}}$ is the path loss exponent and the distance of source-relay link, respectively. $n_{a}^{[R]}(t)$ are the additive white Gaussian noise (AWGN) introduced by the receiving antenna at the relay with mean zero and variance $\sigma_{n_{a}^{[R]}}^{2}$.

The total harvested energy, $E_{R}$ at the relay is

$$
E_{\mathrm{R}}=\eta P_{S}\left|h_{\mathrm{SR}}\right|^{2} d_{\mathrm{SR}}^{-m} \alpha T+0.5 \beta \eta P_{s}\left|h_{\mathrm{SR}}\right|^{2} d_{\mathrm{SR}}^{-m}(1-\alpha) T
$$

where $0<\eta<1$ is the energy conversion efficiency.

After the down conversion, the sampled baseband signal can be written as

$$
y_{\mathrm{R}}(k)=\sqrt{(1-\beta) P_{s} d_{\mathrm{SR}}^{-m}} h_{\mathrm{SR}} s(k)+\sqrt{(1-\beta)} n_{a}^{[\mathrm{R}]}(k)+n_{c}^{[\mathrm{R}]}(k)
$$

where $k$ is the symbol index, $s(k)$ is the sampled information signal from the source, $n_{c}^{[R]}(k)$ is the sampled baseband AWGN due to the conversion with mean zero and variance $\sigma_{n_{c}^{[R]}}^{2}$. 
The relay amplifies and transmits the received signal $x_{\mathrm{R}}$ is given by

$$
x_{\mathrm{R}}(k)=\frac{\sqrt{P_{\mathrm{R}}}}{\sqrt{(1-\beta) P_{s} d_{\mathrm{SR}}^{-m}\left|h_{\mathrm{SR}}\right|^{2}+(1-\beta) \sigma_{n_{a}^{[R]}}^{2}+\sigma_{n_{c}^{[R]}}^{2}}} y_{\mathrm{R}}(k)
$$

where $P_{\mathrm{R}}=\frac{2 E_{\mathrm{R}}}{(1-\alpha) T}$ is the transmitted power from the relay.

\subsubsection{Relay-Destination Transmission}

The sampled received signal at the destination, $y_{D}(k)$, can be expressed as

$$
y_{\mathrm{D}}(k)=\sqrt{P_{\mathrm{R}} d_{\mathrm{RD}}^{-m}} h_{\mathrm{RD}} x_{\mathrm{R}}(k)+n^{[D]}(k)
$$

where $n^{[\mathrm{D}]}(k)$ is AWGN at the destination node with mean zero and variance $\sigma_{n[\mathrm{D}]}^{2}$. Based on the analyses above, the end-to-end SNR of the investigated network with hybrid protocol can be written as

$$
\gamma=\frac{\eta\left(\frac{P_{s}}{N_{0}}\right)^{2} g_{1}^{2} g_{2}\left(\frac{2 \alpha}{1-\alpha}+\beta\right)(1-\beta)}{\eta\left(\frac{P_{s}}{N_{0}}\right) g_{1} g_{2}\left(\frac{2 \alpha}{1-\alpha}+\beta\right)+\left(\frac{P_{s}}{N_{0}}\right) g_{1}(1-\beta)+1}
$$

where $N_{0}$ denotes the mean power of the overall AWGN at relay and destination [10], i.e., $\sigma_{n_{a}^{[R]}}^{2}=$ $\sigma_{n_{c}^{[\mathrm{R}]}}^{2}=\sigma_{n}^{2 \mathrm{D}]}=N_{0} ; g_{1}=\left|h_{\mathrm{SR}}\right|^{2} d_{\mathrm{SR}}^{-m}$ and $g_{2}=\left|h_{\mathrm{RD}}\right|^{2} d_{\mathrm{RD}}^{-m}$.

Since $h_{\mathrm{SR}}$ and $h_{\mathrm{RD}}$ follow Rician fading and Rayleigh fading, respectively, the probability density functions of $g_{1}$ and $g_{2}$ are $[26,27]$

$$
\begin{aligned}
& f_{g_{1}}(x)=\frac{(K+1) e^{-K}}{\lambda_{1}} e^{-\frac{(K+1) x}{\lambda_{1}}} I_{0}\left(2 \sqrt{\frac{K(K+1) x}{\lambda_{1}}}\right) \\
& f_{g_{2}}(x)=\frac{1}{\lambda_{2}} e^{-\frac{x}{\lambda_{2}}}
\end{aligned}
$$

where $K$ denotes the Rician factor; $\lambda_{1}$ and $\lambda_{2}$ are the mean for $g_{1}$ and $g_{2}$, respectively; $I_{0}(\cdot)$ is the zeroth order modified Bessel function of the first kind.

\section{Outage Performance}

This section is devoted to analyzing the outage performance of the investigated network with hybrid protocol. In particular, we first derive an analytical expression for the lower bound outage probability. Based on the derived expression, the expression for upper bound of outage capacity is presented. 
If the end-to-end information rate drops below a predetermined threshold, the outage event happens. On this basis, we definite the outage probability as $P_{\text {out }}=\operatorname{Prob}(\gamma<\tau)$, where $\tau=2^{R}-1$ with the target rate $R[10]$. By means of scaling principle, we have

$$
\begin{gathered}
\gamma<\frac{\tilde{\eta}\left(\frac{P_{s}}{N_{0}}\right)^{2} g_{1}^{2} g_{2}(1-\beta)}{\tilde{\eta}\left(\frac{P_{s}}{N_{0}}\right) g_{1} g_{2}+\left(\frac{P_{s}}{N_{0}}\right) g_{1}(1-\beta)} \\
<\min \left\{\frac{P_{s}(1-\beta)^{2} \tilde{g}_{1}}{N_{0}}, \frac{P_{s} \tilde{\eta}(1-\beta)^{2} \tilde{g}_{1} \tilde{g}_{2}}{N_{0}}\right\}
\end{gathered}
$$

where $\tilde{\eta} \triangleq[2 \alpha /(1-\alpha)+\beta] \eta, \tilde{g}_{1} \triangleq g_{1} /(1-\beta)$ and $\tilde{g}_{2} \triangleq g_{2} /(1-\beta)$.

Based on Equation (9), the outage probability can be further written as

$$
P_{\text {out }} \geq \operatorname{Prob}\left(\frac{P_{s} \tilde{g}_{1}(1-\beta)^{2}}{N_{0}} \min \left\{\tilde{\eta} \tilde{g}_{2} 1\right\}<\tau\right) \triangleq P_{\text {out }}^{\mathrm{LB}}
$$

After obtaining the $P_{\text {out }}^{\mathrm{LB}}$, the upper bound of outage capacity is calculated as

$$
C_{\text {out }}^{\mathrm{UB}}=\frac{(1-\alpha)\left(1-P_{\mathrm{out}}^{\mathrm{LB}}\right) R T}{2} .
$$

Based on Equations (10) and (11), it can be found that the main challenge is to find the closed-form expression for $P_{\text {out }}^{\mathrm{LB}}$, which will be addressed below.

According to the total probability formula, $P_{\text {out }}^{\mathrm{LB}}$ can be expressed as $P_{\text {out }}^{\mathrm{LB}}=M_{1}+M_{2}$, where $M_{1}=\int_{0}^{\frac{1}{\tilde{\eta}}} f_{\tilde{g}_{2}}(y) F_{\tilde{g}_{1}}\left(\frac{\tau}{\hat{c}_{0} \tilde{\eta} y}\right) d y$ and $M_{2}=\int_{\frac{1}{\tilde{\eta}}}^{\infty} f_{\tilde{g}_{2}}(y) \operatorname{Prob}\left(\hat{c}_{0} \tilde{g}_{1}<\tau\right) d y$ with $\hat{c}_{0} \triangleq(1-\beta)^{2} P_{s} / N_{0}$.

Derivation of $M_{1}$. By using the concept of change of variables such as $x=\sqrt{x}$, and the Equation (10) of reference [28], the first term, $M_{1}$, is

$$
\begin{aligned}
M_{1} & =1-e^{-\frac{1-\beta}{\tilde{\eta} \lambda_{2}}}- \\
& \underbrace{\frac{1-\beta}{\lambda_{2}} \int_{0}^{\frac{1}{\tilde{\eta}}} e^{-\frac{(1-\beta) y}{\lambda_{2}}} Q\left(\sqrt{2 K}, \sqrt{\frac{2 \tau(1+K)(1-\beta)}{\hat{c}_{0} \tilde{\eta} \lambda_{1} y}}\right) d y}_{\Xi}
\end{aligned}
$$

where $Q(\cdot, \cdot)$ denotes the first-order Marcum $Q$ function [28].

With the help of integrating by parts for Equation (12) and the equality that $Q(a, \infty)=0$ [28], $\Xi$ can be rewritten as

$$
\begin{aligned}
\Xi & =\underbrace{\int_{0}^{\frac{1}{\tilde{\eta}}} e^{-\frac{(1-\beta) y}{\lambda_{2}}} d Q\left(\sqrt{2 K}, \sqrt{\frac{2 \tau(1+K)(1-\beta)}{\hat{c}_{0} \tilde{\eta} \lambda_{1} y}}\right)}_{\Delta}-Q\left(\sqrt{2 K}, \sqrt{\frac{2 \tau(1+K)(1-\beta)}{\hat{c}_{0} \lambda_{1}}}\right) e^{-\frac{(1-\beta)}{\tilde{\eta} \lambda_{2}}} \\
& \approx \Delta-e^{-\frac{(1-\beta)}{\tilde{\eta} \lambda_{2}}}+\frac{\tau(1+K)(1-\beta)}{\hat{c}_{0} \lambda_{1}} e^{-K-\frac{(1-\beta)}{\tilde{\eta} \lambda_{2}}}
\end{aligned}
$$


where the last approximation is valid at high SNR region since $Q(a, b) \approx 1-0.5 b^{2} e^{\left(-a^{2} / 2\right)}$ holds when $b \rightarrow 0$ [27].

Using the equality that $\frac{\partial Q(a, b)}{\partial b}=-b \exp \left(-\frac{a^{2}+b^{2}}{2}\right) I_{0}(a b)[28]$ and the definition that $\hat{\tau}=\frac{\tau}{\hat{c}_{0}}$, the first term of $\Xi, \Delta$, is

$$
\Delta=\frac{(1+K)(1-\beta)}{\tilde{\eta} \lambda_{1}} e^{-K} \times \underbrace{\hat{\tau} \int_{0}^{\frac{1}{\tilde{\eta}}} \frac{1}{y^{2}} e^{-\frac{(1-\beta) y}{\lambda_{2}}-\frac{\hat{\tau}(1+K)(1-\beta)}{\tilde{\eta} \lambda_{1} y}} I_{0}\left(\sqrt{\frac{4 \hat{\tau} K(1+K)(1-\beta)}{\tilde{\eta} \lambda_{1} y}}\right) d y}_{J}
$$

It is difficult to derive a closed-form expression for $\Delta$ due to the involved zeroth order modified Bessel function of the first kind and complex exponential function. Therefore, we try to find an approximation instead of the closed-form expression at high SNR region in the following. Again, by using the concept of change of variables and the Maclaurin series of exponential function, Equation (14) can be rewritten as

$$
\begin{aligned}
J & =\underbrace{\int_{\tilde{\eta} \hat{\tau}}^{\infty} e^{-\frac{(1+K)(1-\beta) x}{\hat{\eta} \lambda_{1}}} I_{0}\left(\sqrt{\frac{4 K(1+K)(1-\beta) x}{\tilde{\eta} \lambda_{1}}}\right)}_{J_{0}} d x \\
& -\underbrace{\frac{(1-\beta) \hat{\tau}}{\lambda_{2}} \int_{\tilde{\eta} \hat{\tau}}^{\infty} \frac{1}{x} e^{-\frac{(1+K)(1-\beta) x}{\hat{\eta} \lambda_{1}}} I_{0}\left(\sqrt{\frac{4 K(1+K)(1-\beta) x}{\tilde{\eta} \lambda_{1}}}\right) d x}_{J_{1}} \\
& +\underbrace{\sum_{n=2}^{\infty} \frac{\left(\frac{-(1-\beta) \hat{\tau}}{\lambda_{2}}\right)^{n}}{n !} \int_{\tilde{\eta} \hat{\tau}}^{\infty} \frac{1}{x^{n}} e^{-\frac{(1+K)(1-\beta) x}{\hat{\eta} \lambda_{1}}} I_{0}\left(\sqrt{\frac{4 K(1+K)(1-\beta) x}{\tilde{\eta} \lambda_{1}}}\right) d x}_{J_{2}} .
\end{aligned}
$$

Based on (Equation (9), [28]), the first term in (15) can be calculated as

$$
\begin{aligned}
J_{0} & =\frac{\tilde{\eta} \lambda_{1} e^{K}}{(1+K)(1-\beta)} Q\left(\sqrt{2 K}, \sqrt{\frac{2 \hat{\tau}(1+K)(1-\beta)}{\lambda_{1}}}\right) \\
& \approx \frac{\tilde{\eta} \lambda_{1} e^{K}}{(1+K)(1-\beta)}\left(1-\frac{\hat{\tau}(1+K)(1-\beta)}{\lambda_{1}} e^{-K}\right)
\end{aligned}
$$

Based on the equality that $I_{0}(z)=\sum_{k=0}^{\infty} \frac{(z / 2)^{2 k}}{(k !)^{2}}[29]$, we have

$$
J_{1}=\frac{2(1-\beta) \hat{\tau}}{\lambda_{2}} \underbrace{\int_{\sqrt{\tilde{\eta} \hat{\imath}}}^{\infty} \frac{1}{y} e^{-\frac{(1+K)(1-\beta)}{\tilde{\eta} \lambda_{1}}} y^{2}}_{J_{1,0}(\hat{\tau})} d y+\frac{2(1-\beta) \hat{\tau}}{\lambda_{2}} \sum_{k=1}^{\infty} \frac{\left(\sqrt{\frac{K(1+K)(1-\beta)}{\tilde{\eta} \lambda_{1}}}\right)}{(k !)^{2}} \underbrace{\int_{\sqrt{\tilde{\eta} \hat{\imath}}}^{\infty} y^{2 k-1} e^{-\frac{(1+K)(1-\beta)}{\tilde{\eta} \lambda_{1}}} y^{2}}_{J_{1, k}(\hat{\tau})} d y
$$

Using $\int_{u}^{\infty} \frac{e^{-b x^{n}}}{x^{m}} d x=\frac{\Gamma\left(z, b u^{n}\right)}{n b^{z}}$ [29], we have

$$
J_{1,0}(\hat{\tau})=\frac{1}{2} \Gamma\left(0, \frac{(1+K)(1-\beta) \hat{\tau}}{\lambda_{1}}\right) .
$$


By means of $\Gamma(0, x)=-C_{\text {Euler }}-\ln (x)-\sum_{k=1}^{\infty} \frac{(-x)^{k}}{k \cdot k !}[29], J_{1,0}(\hat{\tau})$ can be rewritten as

$$
J_{1,0}(\hat{\tau}) \approx-\frac{1}{2}\left[C_{\text {Euler }}+\ln \left(\frac{(1+K)(1-\beta) \hat{\tau}}{\lambda_{1}}\right)\right]
$$

where the $\Gamma(\cdot, \cdot)$ means upper incomplete gamma function, and Euler's constant $C_{\text {Euler }}=0.5772156649$. Note that the approximation is valid at high SNR region since $\sum_{k=1}^{\infty} \frac{\left(-\frac{(1+K)(1-\beta) \hat{\tau}}{\lambda_{1}}\right)^{k}}{k \cdot k !} \approx 0$ holds when $\hat{\tau} \rightarrow 0$.

Using $\int_{0}^{\infty} y^{2 n+1} e^{-p y^{2}} d y=\frac{n !}{2 p^{n+1}}[29], J_{1, k}(\hat{\tau})$ is given by

$$
J_{1, k}(\hat{\tau})=\frac{(k-1) !}{2\left(\frac{(1+K)(1-\beta)}{\tilde{\eta} \lambda_{1}}\right)^{k}}-\underbrace{\int_{0}^{\sqrt{\tilde{\eta} \hat{\imath}}} y^{2 k-1} e^{-\frac{(1+K)(1-\beta)}{\tilde{\eta} \lambda_{1}}} y^{2}}_{J_{2, k}(\hat{\imath})} d y .
$$

Since $J_{2, k}(\hat{\tau})$ is mathematically intractable, we use Gaussian-Chebyshev quadrature (Gaussian-Chebyshev quadrature is an effective approach to approximate the integral of the given function over a bounded interval, which has been widely used in the filed of performance analysis (see $[13,19]$ for example)) to find an approximation as

$$
J_{2, k}(\hat{\tau}) \approx \frac{\sqrt{\tilde{\eta} \hat{\tau}}}{2} \sum_{i=1}^{N} \frac{\pi}{N} x_{i}^{2 k-1} e^{-\frac{(1+K)(1-\beta)}{\tilde{\eta} \lambda_{1}} x_{i}^{2}} \sin \frac{2 i-1}{2 N} \pi
$$

where $x_{i}=\frac{\sqrt{\tilde{\eta} \hat{\tau}}}{2}\left(1+\cos \frac{2 i-1}{2 N} \pi\right)$, and $N$ is a parameter determining the tradeoff between complexity and accuracy.

Submitting Equations (19)-(21) into Equation (17), $J_{1}$ can be obtained. Through some mathematical manipulations, the third term of Equation (15) can be rewritten as

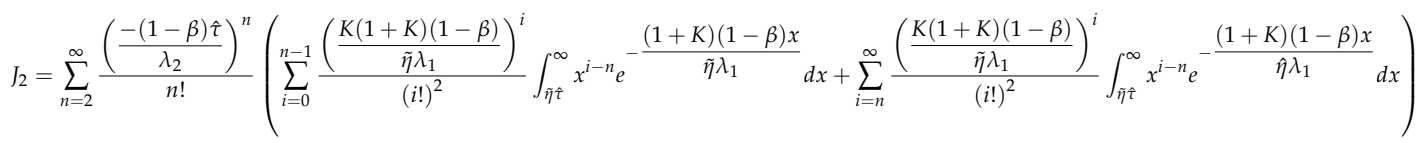

$$
\begin{aligned}
& =\sum_{n=2}^{\infty} \frac{\left(\frac{-(1-\beta) \hat{\tau}}{\lambda_{2}}\right)^{n}}{n !} \sum_{i=0}^{n-2} \frac{\left(\frac{K(1+K)(1-\beta)}{\tilde{\eta} \lambda_{1}}\right)^{i}}{(i !)^{2}} \frac{(-1)^{n-i-1}}{(n-i-1) !}\left(E_{1}\left(\frac{(1+K)(1-\beta) \hat{\tau}}{\lambda_{1}}\right)-e^{-\frac{(1+K)(1-\beta) \hat{\tau}}{\lambda_{1}}} \sum_{j=1}^{n-i-2} \frac{(-1)^{j} j !}{\left(\frac{(1+K)(1-\beta) \hat{t}}{\lambda_{1}}\right)^{j+1}}\right) \\
& +\sum_{n=2}^{\infty} \frac{\left(\frac{-(1-\beta) \hat{\tau}}{\lambda_{2}}\right)^{n}}{n !} E_{1}\left(\frac{(1+K)(1-\beta) \hat{\tau}}{\lambda_{1}}\right)+\sum_{n=2}^{\infty} \frac{\left(\frac{-(1-\beta) \hat{\tau}}{\lambda_{2}}\right)^{n}}{n !}\left(\frac{(1+K)(1-\beta)}{\hat{\eta} \lambda_{1}}\right)^{n-1} \\
& \times \sum_{i=n}^{\infty} \frac{(i-n) ! K^{i}}{(i !)^{2}} e^{-\frac{(1+K)(1-\beta) \hat{\tau}}{\lambda_{1}}} \sum_{m_{1}=0}^{i-n} \frac{((1+K)(1-\beta) \hat{\tau})^{m_{1}}}{\lambda_{1} m_{1} m_{1} !}
\end{aligned}
$$

where the first equality holds from $I_{0}(z)=\sum_{k=0}^{\infty} \frac{\left(\frac{z}{2}\right)^{2 k}}{(k !)^{2}}$ [29]; the second equality is derived from $\Gamma(n, x)=(n-1) ! e^{-x} \sum_{m=1}^{n-1} \frac{x^{m}}{m !}$ [29], $E_{1}(x)=\int_{x}^{\infty} e^{-t} t^{-1} d t$ [29], and $\Gamma(-n, x)=$ $\frac{(-1)^{n}}{n !}\left[E_{1}(x)-e^{-x} \sum_{j=0}^{n-1} \frac{(-1)^{j} j !}{x^{j+1}}\right]^{m=1}$ [30]. Note that $E_{1}(x)=-\operatorname{Ei}(-x)$ and the value of $\operatorname{Ei}(x)$ can be 
calculated by the 'expint' function in MATLAB. Accordingly, by summarizing the above results, $M_{1}$ can be attained.

Derivation of $M_{2}$. Similar as above, using the concept of change of variables and the Equation (10) of reference [28], we can write $M_{2}$ as

$$
\begin{aligned}
M_{2} & =\left(1-Q\left(\sqrt{2 K}, \sqrt{\frac{2 \hat{\tau}(1+K)(1-\beta)}{\lambda_{1}}}\right)\right) e^{-\frac{1-\beta}{\tilde{\eta} \lambda_{2}}} \\
& \approx \frac{2 \hat{\tau}(1+K)(1-\beta)}{\lambda_{1}} e^{-K-\frac{1-\beta}{\tilde{\eta} \lambda_{2}}}
\end{aligned}
$$

where the last approximation holds at high SNR region due to $Q(a, b) \approx 1-b^{2} e^{\left(-a^{2} / 2\right)}$ when $b \rightarrow 0$ [27].

\section{Simulation Results}

Here, numerical simulations are provided to verify our derived results and investigate the efforts of the different system parameters. Unless otherwise stated, the simulation parameters follow [10,23,24], i.e., $\lambda_{1}=\lambda_{2}=1, \eta=1, N_{0}=0.01 \mathrm{~W}, P_{s}=1 \mathrm{~J} / \mathrm{s}, T=1, K=3+\sqrt{12}$, $R=1 \mathrm{bit} / \mathrm{s} / \mathrm{Hz}$. The distances, $d_{\mathrm{SR}}$ and $d_{\mathrm{RD}}$, are normalized [10].

Figure 2 plots the outage capacity with respect to $\beta$ (or $\alpha$ ) for a given $\alpha$ (or $\beta$ ) for the hybrid protocol. The theoretical results are obtained from Equation (11). One can see that the concurrence between the simulated results and the theoretical results verify the theoretical analyses on the outage probability and the outage capacity. One can also see that different parameters of hybrid protocol achieve different outage capacity. This implies that a good selection of parameters of hybrid protocol has a significant impact on the outage capacity, and that the near-optimal parameters of hybrid protocol can be obtained through maximizing Equation (11).

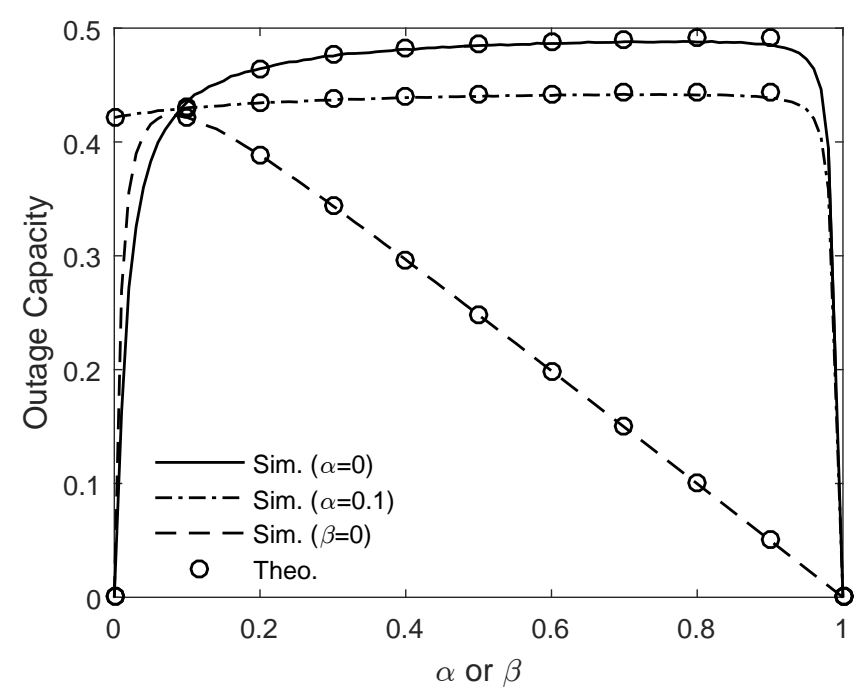

Figure 2. Outage Capacity against power splitting (PS)/time switching (TS) ratio.

Figure 3 compares the outage capacity of the optimal hybrid protocol, the optimal PS protocol, and the optimal TS protocol under different $R$. The parameters of the optimal PS protocol, the optimal TS protocol, and the optimal HR protocol are obtained by maximizing the outage capacity and its optimal parameters are plotted at the lower part of Figure 3. One observation is that the outage capacity increases to the peak value first then decreases for each protocol. Another observation is that the hybrid protocol always achieves a higher capacity than both PS protocol and TS protocol. In particular, there is only a marginal difference between the hybrid protocol and PS protocol in terms of outage 
capacity when $1 \leq R \leq 3$, and the TS protocol can be regarded as a near-optimal hybrid protocol when $R \geq 5$. This observation reveals that the hybrid protocol can be reduced to PS protocol or TS protocol at some certain system parameters. This revelation is highly beneficial to reduce the complexity of hybrid protocol while maintaining the required outage performance. Finally, we investigate the impacts of energy conversion efficiency and Rician $K$-factor on the investigated network with optimal hybrid protocol in Figure 4. It can be seen that a low energy conversion efficiency leads to a low outage capacity. This is because both larger TS ratio and PS ratio are required to harvest enough energy at a low $\eta$, resulting in a low capacity. Besides, it can be drawn that a larger value of $K$ achieves a higher outage capacity.

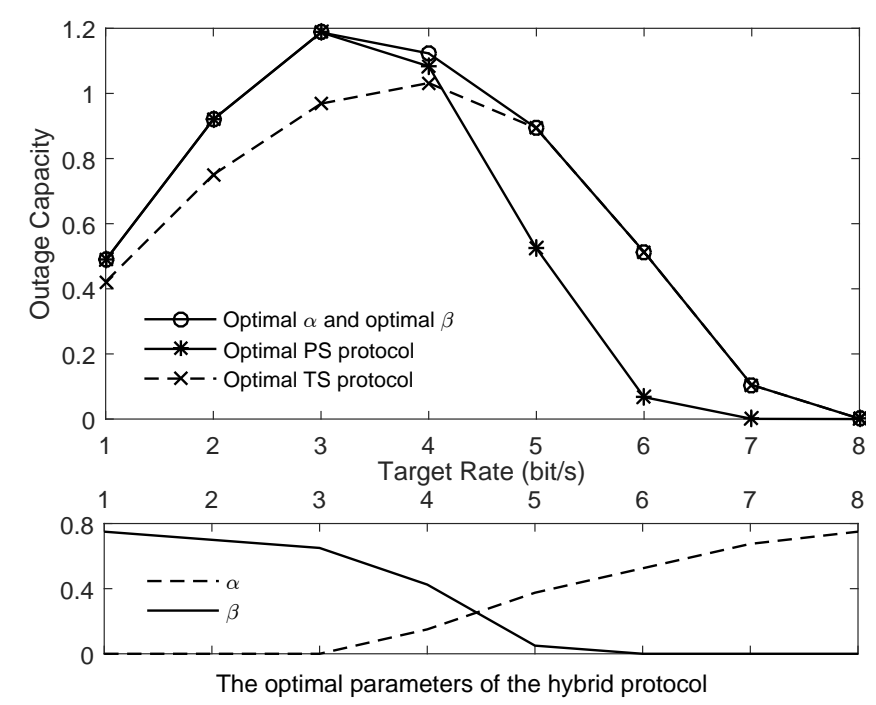

Figure 3. Outage Capacity against $R$ and the optimal parameters of the hybrid protocol.

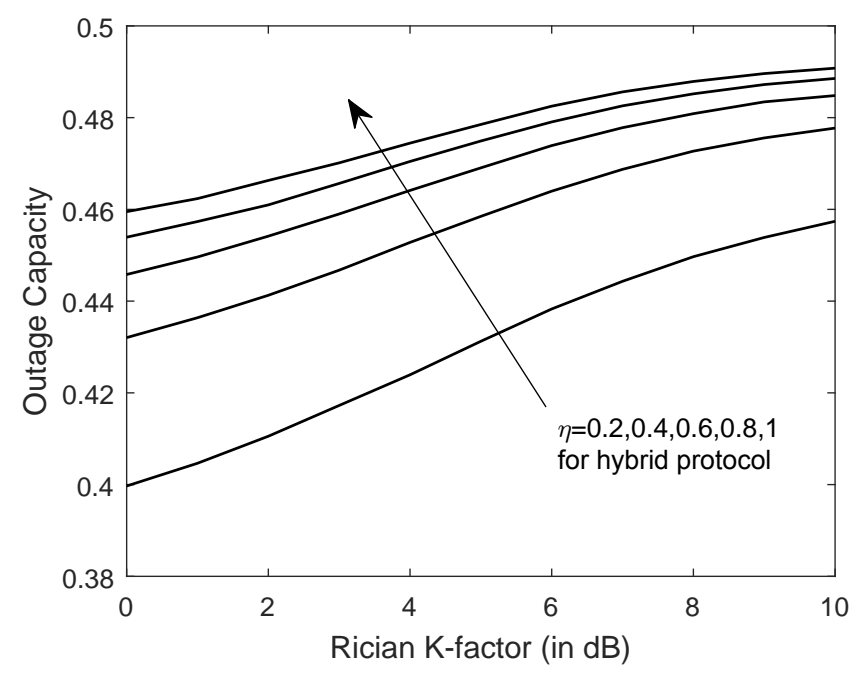

Figure 4. Outage Capacity against $K$ and $\eta$.

\section{Conclusions}

In this paper, the outage performance of an hybrid protocol for AF relaying over asymmetric fading channels has been studied. In particular, the expressions of the outage probability and outage capacity have been derived and verified. The effects of the parameters of the hybrid protocol on the outage capacity have been investigated. The significance of our work is two-fold. First, our work provides theoretical analyses and simulation results to characterize the outage performance of the hybrid protocol based AF EH relaying over asymmetric fading channels. These contributions not only 
reveal the importance of the parameters selection for hybrid protocol, but also provide a solution to obtain the optimal parameters of the hybrid protocol in the investigated networks. Second, we show that a larger Rician factor and energy conversion efficiency are desirable for the investigated network.

Finally, we point out two possible future works. First, it is interesting to study the performance analysis of hybrid protocol based multiple relay network over asymmetric fading channels. Second, the linear energy harvesting model considered in this paper can be extended to the non-linear model, making our considered network close to practical scenarios.

Author Contributions: Formal analysis, X.S. and G.L.; Funding acquisition, G.L.; Investigation, L.S.; Methodology, Y.Y.; Supervision, G.L.; Writing—original draft, X.S.; Writing—review \& editing, G.L., L.S. and Y.Y.

Funding: This work was supported by the the Natural Science Foundation of China $(61701399,61501371)$, the Science and Technology Innovation Team of Shaanxi Province for Broadband Wireless and Application (2017KCT-30-02), the Research Program of Education Bureau of Shaanxi Province (17JK0699).

Conflicts of Interest: The authors declare no conflict of interest.

\section{References}

1. Guo, W.; Zhou, S.; Chen, Y.; Wang, S.; Chu, X.; Niu, Z. Simultaneous information and energy flow for IoT relay systems with crowd harvesting. IEEE Commun. Mag. 2016, 54, 143-149. [CrossRef]

2. Ciuonzo, D.; Rossi, P.S.; Dey, S. Massive MIMO channel-aware decision fusion. IEEE Trans. Signal Process. 2015, 63, 604-619. [CrossRef]

3. Ciuonzo, D.; Romano, G.; Rossi, P.S. Channel-aware decision fusion in distributed MIMO wireless sensor networks: Decode-and-Fuse vs. Decode-then-Fuse. IEEE Trans. Wirel. Commun. 2012, 11, $2976-2985$. [CrossRef]

4. Shirazinia, A.; Dey, S.; Ciuonzo, D.; Rossi, P.S. Massive MIMO for decentralized estimation of a correlated source. IEEE Tran. Signal Process. 2016, 64, 2499-2512. [CrossRef]

5. Jiang, F.; Chen, J.; Swindlehurst, A.L.; López-Salcedo, J.A. Massive MIMO for wireless sensing with a coherent multiple access channel. IEEE Trans. Signal Process. 2015, 63, 3005-3017. [CrossRef]

6. Ding, G.; Gao, X.; Xue, Z.; Wu, Y.; Shi, Q. Massive MIMO for distributed detection with transceiver impairments. IEEE Trans. Veh. Technol. 2018, 67, 604-617. [CrossRef]

7. Tsiropoulou, E.E.; Mitsis, G.; Papavassiliou, S. Interest-aware energy collection \& resource management in machine to machine communications. Ad Hoc Netw. 2018, 68, 48-57.

8. Perera, T.D.P.; Jayakody, D.N.K.; Sharma, S.K.; Chatzinotas, S.; Li, J. Simultaneous wireless information and power transfer (SWIPT): Recent advances and future challenges. IEEE Commun. Surv. Tutor. 2018, 20, 264-302. [CrossRef]

9. Huang, Y.; Liu, M.; Liu, Y. Energy-efficient SWIPT in IoT distributed antenna systems. IEEE Internet Things J. 2018, 5, 2646-2656. [CrossRef]

10. Nasir, A.A.; Zhou, X.; Durrani, S.; Kennedy, R.A. Relaying protocols for wireless energy harvesting and information processing. IEEE Trans. Wirel. Commun. 2013, 12, 3622-3636. [CrossRef]

11. Ye, Y.; Li, Y.; Zhou, F.; Al-Dhahir, N.; Zhang, H. Power splitting-based swipt with dual-hop DF relaying in the presence of a direct link. IEEE Syst. J. 2018, 1-5. [CrossRef]

12. Lee, H.; Song, C.; Choi, S.H.; Lee, I. Outage probability analysis and power splitter designs for SWIPT relaying systems with direct link. IEEE Commun. Lett. 2017, 21, 648-651. [CrossRef]

13. Ye, Y.; Li, Y.; Wang, D.; Zhou, F.; Hu, R.Q.; Zhang, H. Optimal transmission schemes for DF relaying networks using SWIPT. IEEE Trans. Veh. Technol. 2018, 67, 7062-7072. [CrossRef]

14. Atapattu, S.; Jiang, H.; Evans, J.; Tellambura, C. Time-switching energy harvesting in relay networks. In Proceedings of the 2015 IEEE International Conference on Communications (ICC), London, UK, 8-12 June 2015; pp. 5416-5421.

15. Rabie, K.M.; Salem, A.; Alsusa, E.; Alouini, M.S. Energy-harvesting in cooperative AF relaying networks over log-normal fading channels. In Proceedings of the 2016 IEEE International Conference on Communications (ICC), Kuala Lumpur, Malaysia, 22-27 May 2016; pp. 1-7.

16. Ding, H.; da Costa, D.B.; Wang, X.; Dias, U.S.; de Sousa, R.T.; Ge, J. On the effects of LOS path and opportunistic scheduling in energy harvesting relay systems. IEEE Trans. Wirel. Commun. 2016, 15, 8506-8524. [CrossRef] 
17. Rabie, K.M.; Adebisi, B.; Alouini, M.S. Wireless power transfer in cooperative DF relaying networks with log-normal fading. In Proceedings of the 2016 IEEE Global Communications Conference (GLOBECOM), Washington, DC, USA, 4-8 December 2016; pp. 1-6.

18. Ye, J.; Lei, H.; Liu, Y.; Pan, G.; da Costa, D.B.; Ni, Q.; Ding, Z. Cooperative communications with wireless energy harvesting over Nakagami- $m$ fading channels. IEEE Trans. Commun. 2017, 65, 5149-5164. [CrossRef]

19. Ye, Y.; Li, Y.; Wang, D.; Lu, G. Power splitting protocol design for the cooperative NOMA with SWIPT. In Proceedings of the 2017 IEEE International Conference on Communications (ICC), Paris, France, 21-25 May 2017; pp. 1-5.

20. Yan, J.; Liu, Y. A dynamic SWIPT approach for cooperative cognitive radio networks. IEEE Trans. Veh. Technol. 2017, 66, 11122-11136. [CrossRef]

21. Atapattu, S.; Evans, J. Optimal energy harvesting protocols for wireless relay networks. IEEE Trans. Wirel. Commun. 2016, 15, 5789-5803. [CrossRef]

22. Pilanawithana, B.; Atapattu, S.; Evans, J. Energy allocation and energy harvesting in wireless relay networks with hybrid protocol. In Proceedings of the GLOBECOM 2017-2017 IEEE Global Communications Conference, Singapore, 4-8 December 2017; pp. 1-6.

23. Tao, R.; Salem, A.; Hamdi, K.A. Adaptive relaying protocol for wireless power transfer and information processing. IEEE Commun. Lett. 2016, 20, 2027-2030. [CrossRef]

24. Zhao, F.; Lin, H.; Zhong, C.; Hadzi-Velkov, Z.; Karagiannidis, G.K.; Zhang, Z. On the capacity of wireless powered communication systems over rician fading channels. IEEE Trans. Commun. 2018, 66, $404-417$. [CrossRef]

25. Ye, Y.; Li, Y.; Shi, L.; Hu, R.Q.; Zhang, H. Improved hybrid relaying protocol for DF relaying in the presence of a direct link. IEEE Wireless Commun. Lett. 2018. [CrossRef]

26. Suraweera, H.A.; Karagiannidis, G.K.; Smith, P.J. Performance analysis of the dual-hop asymmetric fading channel. IEEE Trans. Wirel. Commun. 2009, 8, 2783-2788. [CrossRef]

27. Ding, H.; Ge, J.; da Costa, D.B.; Guo, Y. Outage analysis for multiuser two-way relaying in mixed rayleigh and rician fading. IEEE Commun. Lett. 2011, 15, 410-412. [CrossRef]

28. Nuttall, A.H. Some integrals involving the q-function. IEEE Trans. Inform. Theory 1972, 21, 95-96. [CrossRef]

29. Gradshteyn, I.S.; Ryzhik, I.M. In table of integrals, series, and products. Math. Comput. 1981, 20, C1157-C1160. [CrossRef]

30. Abramowitz, M.; Stegun, I.A.; Romain, J.E. Handbook of Mathematical Functions, with Formulas, Graphs, and Mathematical Tables; Dover Publications: Mineola, NY, USA, 1972.

(C) 2019 by the authors. Licensee MDPI, Basel, Switzerland. This article is an open access article distributed under the terms and conditions of the Creative Commons Attribution (CC BY) license (http://creativecommons.org/licenses/by/4.0/). 